Prostate cancer has the second highest incidence among malignant tumors in men in Poland, and the first in the USA. Currently, medical reports state that incidentally non-diagnosed prostate cancer is present in about $30-46 \%$ of men over the age of fifty.

Tumor angiogenesis has significant implications in the diagnosis and treatment of various solid tumors. With fast, multi-slice CT scanners and their ability of qualitative and quantitative analysis of tumor angiogenesis, CT perfusion has been the subject of extensive investigation in the past twenty years.

Tumor angiogenesis is characterized morphologically by an increase in the number of blood vessels including new capillaries, capillary sprouts, nonendothelialized capillaries and arteriovenous shunts.

It is stated in the literature that pathological vessel density within prostate cancer is one of the factors determining the extent of the tumor - whether the tumor is confined to the prostate gland or extends beyond the prostate capsule. It was proved that the density of pathological vessels is higher in patients with tumors beyond the prostate capsule. Initial publications show validity of functional imaging, such as perfusion CT in prostate cancer staging before a surgical procedure. This examination can also show excessive density of pathological vessels within the prostate gland according to increased blood volume (BV) and blood flow (BF) and pathologically increased permeability of the vessels (PS). Vessel abnormalities in the examined area are also indicated by shorter mean transit time (MTT).

Key words: prostate cancer, neoangiogenesis, perfusion CT.

\section{Neoangiogenesis in prostate cancer}

\author{
Elżbieta Łuczyńska, Joanna Anioł
}

Department of Diagnostic Imaging, Maria Skłodowska-Curie Memorial Cancer Center and Institute of Oncology, Cracow Branch, Krakow, Poland

\section{Introduction}

Prostate cancer is the second most frequently occurring malignant tumor in men in Poland, and the first in the USA. According to the literature data in the year 2007 in the USA 218890 new prostate cancer cases were diagnosed and 27050 men died due to that disease. Currently, medical reports state that incidental non-diagnosed prostate cancer is present in about $30-46 \%$ of men over the age of fifty.

Traditional diagnostic methods have limitations both in diagnostics and in prostate cancer staging. These limitations are due to poor differentiation of cancer from adjacent normal tissues and from benign prostate hyperplasia (BPH).

Prostate cancer screening is controversial. Prostate-specific antigen (PSA) serum level and digital rectal examination (DRE) are currently accepted standard examination methods in cancer screening. Because of low sensitivity and low specificity in prostate cancer detection, transrectal ultrasound (TRUS) is not considered as a screening method. Core needle biopsy under TRUS guidance in patients with increased PSA serum level or abnormalities found in DRE is an accepted method in prostate cancer diagnostics. Although core needle biopsy improves diagnosis, it provides limited information about neoplastic process extent, caused by the lack of correlation between number of focal lesions and their localization, and actual cancer staging.

Hypoxia present inside the growing tumor is considered as an angiogenic factor, increasing pathologic vessel density and allowing for growth of solid lesions. Prostate cancer demonstrates a significant increase in pathologic vessels in relation to normal prostate tissue. Similar to other organs, pathologic vessel density within the prostate is a predictive factor for tumor malignancy [1].

Perfusion examinations in prostate cancer were primarily used in ultrasound and MRI; a few recent literature data also describe perfusion application in CT.

Proper staging of the cancer is necessary before starting therapy. Prostate cancer staging is evaluated on the basis of per rectum clinical exam, PSA level in blood serum, histopathological report after core needle biopsy of the prostate gland (Gleason score), and on the basis of imaging method results (transrectal ultrasound, magnetic resonance imaging and computed tomography). Proper staging if the cancer is organ confined or extends through the prostate capsule is important in qualification for the best therapy method. Prostate cancer extending through the prostate capsule is a bad prognostic factor.

Tumor angiogenesis has significant implications in the diagnosis and treatment of various solid tumors. With the advent of fast, multi-slice CT scanners and their ability of qualitative and quantitative analysis of tumor angiogenesis, CT perfusion has been a subject of extensive investigation in the past twenty years. The fundamental bases for CT imaging of angiogenesis are both the transport by blood flow of intravenously administered iodinated contrast material to tissue and the exchange by diffusion of these contrast molecules between the intravascular space and the extravascular interstitial space. With current, fast CT scanners both tissue and vascular enhancement can be measured and traced over time at small time intervals to allow detailed modeling of the distribution of contrast agent in tissue. Both compartmental and 
distributed parameter models for contrast transport and exchange have been developed to quantify from the CT data the following angiogenesis related parameters: tissue blood flow, blood volume, mean transit time, and capillary permeability surface area product.

\section{Neoangiogenesis}

Tumor angiogenesis is the process by which new blood vessels are formed from the existing vessels to promote tumor growth. Angiogenesis is essential for tumor growth and tumor metastasis, but tumor genesis or malignant transformation of the tumor is not dependent on angiogenesis. Angiogenesis is a complex process that is mediated by several angiogenic and antiangiogenic factors produced by the tumor cells, the blood and the stroma of the host tissue. The balance in the production of these factors predicts when angiogenesis will develop; angiogenesis develops when the pro-angiogenic factors overcome anti-angiogenic factors. This process includes endothelial proliferation, breakdown of basement membranes of the capillaries, endothelial cell migration into the extravascular space in the stroma, formation of capillary tubes, communication with the postcapillary venules and blood flow through the new blood vessels.

Tumor angiogenesis is characterized morphologically by an increase in the number of blood vessels including new capillaries, capillary sprouts, non-endothelialized capillaries and arteriovenous shunts. Some of these vessels are malfunctioned. They tend to be leaky, allowing large molecules such as plasma protein to enter the stroma to form the matrix for the new vessels. They lack smooth muscle and innervation, and can be easily compressed. What is more, the blood components within the tumor may also alter the blood flow as they become adhering and aggregating to the vessel wall. These changes in the tumor vessels make physiologic and hemodynamic changes of blood flow more complex and heterogeneous among various regions and various types of tumors. In the regions where tumor growth is active, such as at the periphery of the tumors, and where new blood ves-

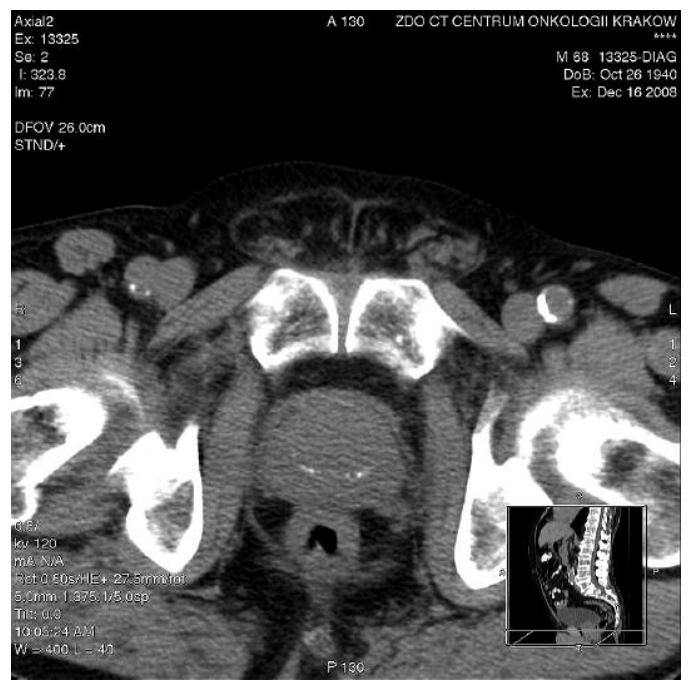

Fig. 1. Computed tomography of the prostate. Pilot scan before contrast injection. Large prostate without pathological lesions visible on classic CT scan. Calcifications in the central zone sels develop good communications with veins or with arterio-venous shunts, there is evidence that blood flow would increase [2].

In the regions where endothelial cells have high vascular permeability, interstitial pressure would increase and can compress capillary sprouts and small capillaries. In those regions blood flow could decrease and could result in tissue hypoxia and necrosis.

Density of the pathological vessels within the prostate gland is strictly correlated with tumor presence, metastases, stage of extension and survival possibilities. Qualitative analysis of pathological vessels (angiogenesis) and their density can provide a number of data for significant therapeutic decisions $[3,4]$.

\section{Computed tomography in prostate cancer staging}

The prostate gland in $\mathrm{CT}$ is visible as a smoothly demarcated structure located at the bottom of the pelvis posteriorly to the pubic symphysis and anteriorly to the rectum, below the urinary bladder. After contrast administration, the prostate transitional zone is brightly enhanced in comparison to the rest of glandular parenchyma. This enhancement is characteristic for benign prostate hyperplasia (BPH) (Fig. 1).

Prostate cancer is not visible in CT examination. Prostate cancer can be visible in CT only in case of prostate capsule pouching or deformation of the external borderline of the gland after contrast administration.

At the beginning of the 1990s helical CT improved the position of tomography in diagnostics of the human body and consolidated CT as a modality for abdominal and pelvic cavity imaging. In the middle of the 1990s double-detector tomography was introduced, and soon after there was the advent of multi-detector CT (MDCT). In MDCT application examination time is shorter, which allows thin slices to be obtained. That results in collecting data from a large volume and later transformation of the picture in the required anatomical plane. Multi-detector CT covering a large area of the body makes multi-phase imaging during administration of contrast medium possible, enabling organ evaluation considering its vascularity and perfusion.

Improvement of CT scanners means that we can make morphological and functional pictures of the body. Morphological pictures show anatomy and pathology of the organ. Functional techniques allow us to see the organ function and pathological processes in tumors, for example angiogenesis.

During intravenous administration of the contrast medium, structures within the scanning area show contrast enhancement, which is correlated with iodinated medium density in the tissue. In the first phase, immediately after intravenous injection of contrast material (the first 45-60 s), the contrast material is visible as predominantly intravascular. Delivering the contrast material to the tissue in this phase depends on cardiac output and circulating blood volume. As time progresses, increasing amounts of contrast material pass into the extravascular space until equilibrium is reached, whereby the rate at which contrast material passes from the vascular to the extravascular space is balanced by the rate at which it returns to the vascular system from the extravascular space. In tumors, significant return of contrast mate- 
rial into the intravascular space can be observed within 2 min. Thus, measurement of vascular permeability requires images to be acquired for up to 2 to $10 \mathrm{~min}$ after administration of contrast medium.

The basis of neoangiogenesis evaluation in CT is not only assessment of blood flow in vessels after administration of contrast material but also contrast molecules' diffusion from the intravascular to the extravascular space.

There is an increasing number of publications reporting a correlation between contrast enhancement parameters and histological measurements of angiogenesis, such as microvessel density (MVD), validating the use of perfusion CT as a marker of angiogenesis.

Li et al. reported a correlation between angiogenesis and CT perfusion in patients with peripheral lung cancer. Assignies et al. found a similar correlation in patients with endocrine tumors of the pancreas. Feng et al. reported a correlation between density of pathological vessels and $\mathrm{CT}$ perfusion in patients with rectal carcinomas [5-7].

With current, fast CT scanners both tissue and vascular enhancement can be measured and traced over time at small time intervals to allow detailed modeling of the distribution of contrast agent in tissue. Both compartmental and distributed parameter models for contrast transport and exchange have been developed to quantify from the CT data the following angiogenesis related parameters: tissue blood flow, blood volume, mean transit time, and capillary permeability surface area product.

\section{Definition of terms and their relationship}

Blood flow - this is the volume flow rate of blood through the vasculature in the tissue region. It is usually expressed in units of $\mathrm{ml} / \mathrm{min}$ per $100 \mathrm{~g}$ of tissue. Blood flow measured with CT using the methodology discussed earlier includes flow in large vessels, arterioles, capillaries, venules and veins. Flow in arterio-venous shunts should also be included.

Blood volume - this is the volume of blood within the vasculature in a tissue region that is actually flowing. As in the case of blood flow, blood volume includes blood in large vessels, arterioles, capillaries, venules and veins. Any stagnant pool of blood is not included in the blood volume. It is measured in units of $\mathrm{ml}$ per $100 \mathrm{~g}$ of tissue.

Mean transit time (MTT) - this is the average time taken by blood elements to traverse the vasculature from the arterial end to the venous end. If the perfusion pressure is high, blood elements are travelling at a higher velocity, resulting in a shorter mean transit time than when the perfusion pressure is low. In this sense, mean transit time is a surrogate measure of perfusion pressure. Mean transit time is usually measured in seconds.

Capillary permeability surface area product (PS) - the product of permeability and blood concentration of the solute gives the unidirectional diffusional flux from blood to interstitial space per unit surface area of capillary endothelium. Permeability surface area product is the product of permeability and the total surface area of capillary endothelium in a unit mass of tissue (usually $100 \mathrm{~g}$ of tissue) and hence is the total diffusional flux across all capillaries. It is measured

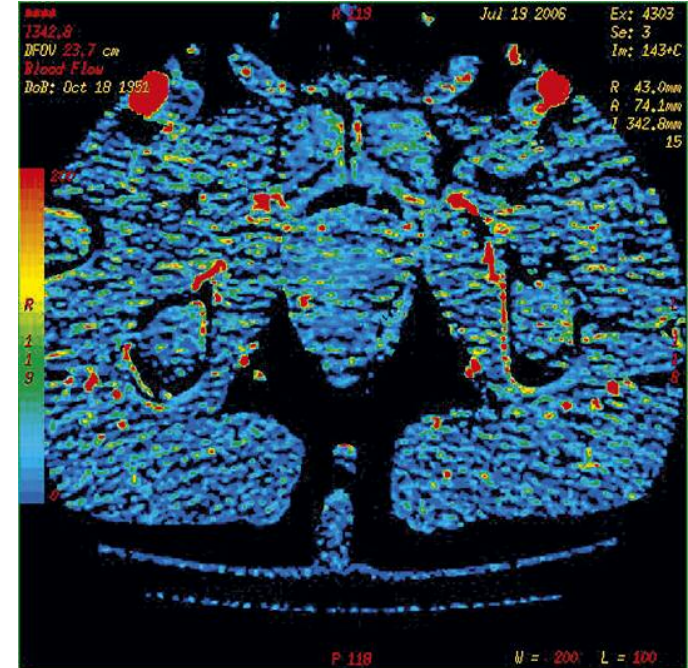

Fig. 2. Computed tomography of the prostate - perfusion option. Parametric map of blood flow. Focal lesion of pathologically high blood flow in the right peripheral zone. Lesion extends outside prostate through the capsule, goes into the central zone at the right site

in units of $\mathrm{ml} / \mathrm{min}$ per $100 \mathrm{~g}$ of tissue. Expressed in the stated units, PS can be interpreted as that the unidirectional flux of solutes from blood plasma to the interstitial space is equivalent to the complete transfer of all the solutes in PS $\mathrm{ml}$ of blood per minute to the interstitial space. Permeability surface area product is implicitly tied to the experimental setup in which a semi-permeable membrane is separating two solvents containing a solute at different concentrations. It is equal to the diffusional flux of solute across the whole semipermeable membrane (capillary endothelium) when the solvent - blood - is stationary with respect to the membrane. For the more physiological case of blood flowing through capillaries, the unidirectional flux of blood-borne solutes through all capillaries is dependent on blood flow and PS [8].

Due to the recent development of MDCT and the possibility of obtaining color-coded perfusion maps, which correspond to classic CT images, we are able to assess prostate cancer using this method. Prostate cancer in perfusion examination is characterized by increased BF, BV and PS and decreased MTT [9] (Figs. 2-5). Increased BF in the tumor in comparison to normal tissue is caused by opening arteriovenous shunts in the tumor. Pathological connections between arteries and veins cause lower resistance for blood flow and induct higher flow between pathological microvessels. Increasing BV causes decreasing MTT in those vessels.

Higher BV inside the tumor in comparison to normal tissue can be explained as an increased number of microvessels caused by neoangiogenesis. Increased PS in the tumor in comparison to normal tissue is correlated with higher permeability of endothelium in new vessels; they have different structure than in normal tissue. Perfusion CT examinations show that perfusion is higher in very aggressive tumors in comparison to lesions of lower malignancy [10, 11].

In conclusion, prostate cancer does not belong to the group of tumors of well-developed vascularity. It was proved, how- 


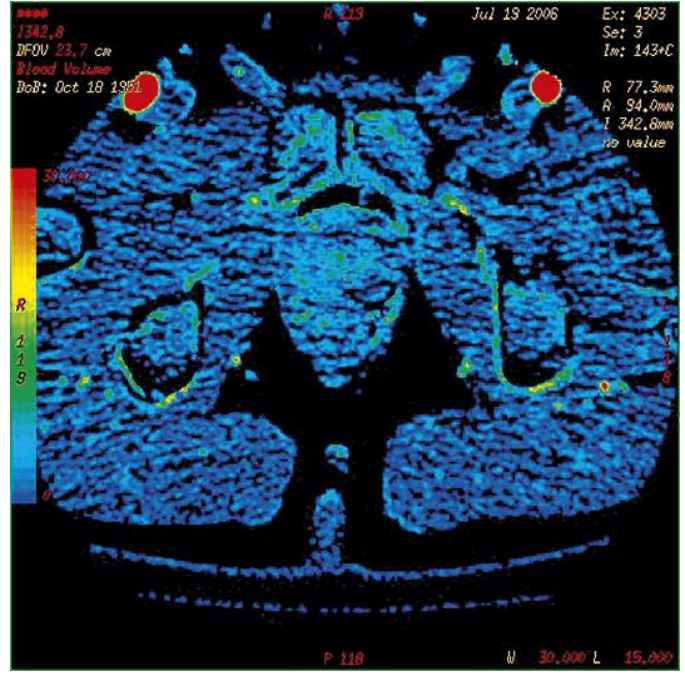

Fig. 3. Computed tomography of the prostate - perfusion option. Parametric map of blood volume. Focal lesion of pathologically high blood volume in the right peripheral zone

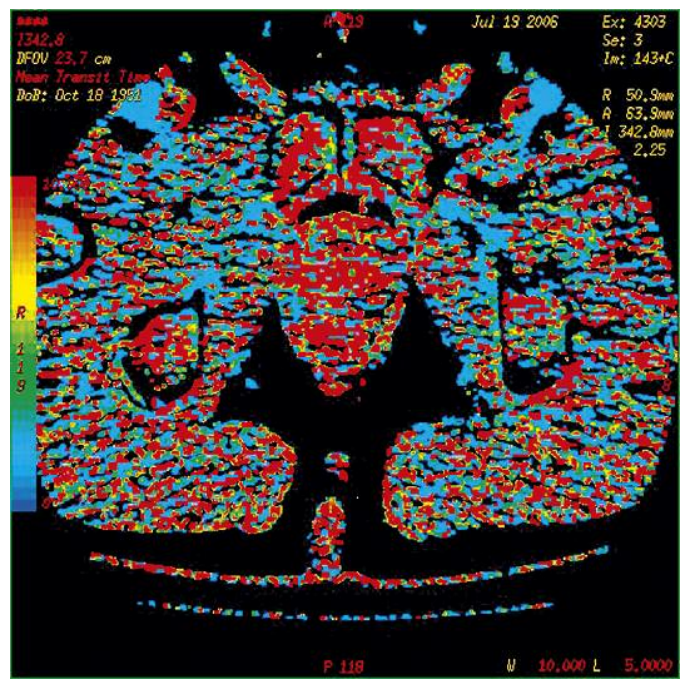

Fig. 5. Computed tomography of the prostate - perfusion option. Parametric map of mean transit time. Focal lesion of pathological angiogenesis in the right peripheral zone

ever, that increase in pathological vessel density within the prostate cancer compared with normal prostate tissue is significant [11]. The conclusion of the highest density of vessels in poorly differentiated prostate cancer results from the observation [12]. It is stated in the literature that pathological vessel density within prostate cancer is one of the factors determining the extent of the tumor - whether the tumor is confined to the prostate gland or extends beyond the prostate capsule. It was proved that the density of pathological vessels is higher in patients with tumors beyond the prostate capsule [13]. Initial publications show significance of functional imaging, such as perfusion CT in prostate cancer staging before a surgical procedure. This examination can also show excessive density of pathological vessels within the prostate gland according to increased BV and BF and pathologically increased permeability of the vessels (PS). Vessel abnormalities in the examined area are also indicated by shorter MTT.

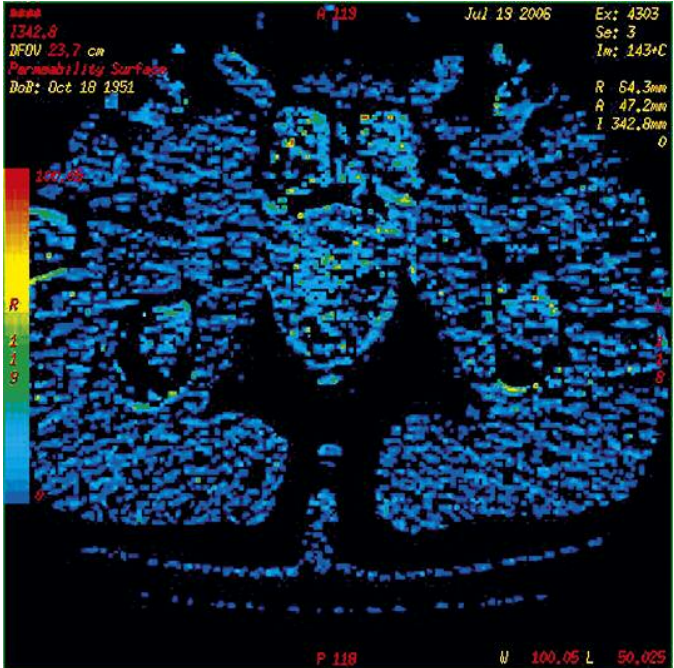

Fig. 4. Computed tomography of the prostate - perfusion option Parametric map of permeability surface. Focal lesion of pathological angiogenesis in the right peripheral zone

Bostwick states the importance of vessel density within the tumor in prostate cancer evaluation (in case of cancer confined to the gland). For example, probability of tumor extension beyond the capsule is $93 \%$ for a patient with a PSA level of $8 \mathrm{ng} / \mathrm{ml}$, Gleason score of 8 after core biopsy, and high vascular density found in histopathological examination [14].

Literature data indicate that the number and size of foci found in prostate perfusion examination is higher in patients with a high Gleason score (8 and more) and with a larger tumor volume in patients with prostate adenocarcinoma. According to other publications (Thomas Jefferson University) perfusion CT is important in patients with high volume and high progression of prostate cancer; however, it is not useful for staging most prostate cancer patients. This is related to the fact that prostate cancer, unlike other solid tumors, commonly grows multifocally, often spreads beyond the capsule, and can also grow into an irregular shape along the anatomical prostate capsule. Such histologic cancer growth restricts the ability for tumor visualization not only in perfusion $\mathrm{CT}$, but also in other methods, such as ultrasound or MRI $[15,16]$.

The authors declare no conflict of interest.

\section{References}

1. Lee TY, Purdie TG, Stewart E. CT imaging of angiogenesis. Q J Nucl Med 2003; 47: 171-87.

2. Folkman J, Watson K, Ingber D, Hanahan D. Induction of angiogenesis during the transition from hyperplasia to neoplasia. Nature 1989; 339: $58-61$

3. Miles KA. Tumour angiogenesis and its relation to contrast enhancement on computed tomography: a review. Eur J Radiol 1999; 30: 198-205.

4. Miles KA. Perfusion CT for the assessment of tumour vascularity: which protocol? Br J Radiol 2003; 76 Spec No 1: S36-42.

5. Li Y, Yang ZG, Chen TW, Chen HJ, Sun JY, Lu YR. Peripheral lung carcinoma: correlation of angiogenesis and first-pass perfusion parameters of 64-detector row CT. Lung Cancer 2008; 61: 44-53. 
6. d'Assignies G, Couvelard A, Bahrami S, et al. Pancreatic endocrine tumors: tumor blood flow assessed with perfusion CT reflects angiogenesis and correlates with prognostic factors. Radiology 2008; 250: 407-16.

7. Feng ST, Sun CH, Li ZP, et al. Evaluation of microvessel density and vascular endothelial growth factor in colorectal carcinoma with 64-multidetector-row CT perfusion imaging. Zhonghua Wei Chang Wai Ke Za Zhi 2008; 11: 537-41.

8. Miles KA. Functional computed tomography in oncology. Eur J Cancer 2002; 38: 2079-84.

9. Miles KA, Griffiths MR. Perfusion CT: a worthwhile enhancement? Br J Radiol 2003; 76: 220-31.

10. Dugdale PE, Miles KA, Bunce I, Kelley BB, Leggett DA. CT measurement of perfusion and permeability with lymphoma masses and ability to assess grade, activity, and chemotherapeutic response. J Comput Assist Tomogr 1999; 23: 540-7.

11. Sahani DV, Holalkere NS, Mueller PR, Zhu AX. Advanced hepatocellural carcinoma: CT perfusion of liver tumor tissue - initial experience. Radiology 2007; 243: 736-43.

12. Bigler SA, Deering RE, Brawer MK. Comparison of microscopic vascularity on begin and malignant prostate tissue. Human Pathol 1993; 24: 220-6.

13. Weidner N, Carroll PR, Flax J, Blumenfeld W, Folkman J. Tumor angiogenesis correlates with metastasis in invasive prostate carcinoma. Am J Pathol 1993; 143: 401-9.

14. Bostwick DG, Wheeler TM, Blute M, et al. Optimized microvassel density analysis improves prediction of cancer stage from prostate needle biopsies. Urology 1996; 48: 47-57.

15. Łuczyńska E, Anioł J, Szczudło J, et al. Perfusion of prostate cancer: correlation between $\mathrm{p}$-CT and whole - mount pathology - case report. Pol J Radiology 2006; 71: 70-3.

16. Ives EP, Burke MA, Edmonds PR, Gomella LG, Halpern EJ. Quantitative computed tomography perfusion of prostate cancer: correlation with whole-mount pathology. Clin Prostate Cancer 2005; 4: 109-12.

\section{Address for correspondence}

\section{Elżbieta łuczyńska}

Department of Diagnostic Imaging

Maria Skłodowska-Curie Memorial Cancer Center

and Institute of Oncology

Krakow Branch

Garncarska 11

31-115 Krakow, Poland

e-mail: z5luczyn@cyfronet.pl

Submitted: $\quad 28.01 .2011$

Accepted: $\quad 8.05 .2012$ 